\title{
Distribution of digits in integers: fractal dimensions and zeta functions
}

\author{
by
}

L. Olsen (St. Andrews)

1. Introduction and statement of results. Let $N \geq 2$ be a fixed positive integer. For a positive integer $n \in \mathbb{N}$, let

$$
n=d_{0}(n)+d_{1}(n) N+d_{2}(n) N^{2}+\ldots+d_{\gamma(n)}(n) N^{\gamma(n)},
$$

where $d_{i}(n) \in\{0,1, \ldots, N-1\}$ and $d_{\gamma(n)}(n) \neq 0$, denote the $N$-ary expansion of $n$.

The purpose of this paper is to introduce a very general class of subsets of $\mathbb{N}$ defined in terms of the asymptotic behaviour of the digits in the $N$-ary expansion of the integers, and to study the "fractal" dimensions and zeta functions associated with these sets. In particular, we compute the "fractal" dimensions of the set of positive integers $n$ such that each digit $i$ in $n$ appears with frequence $p_{i}$ for some probability vector $\mathbf{p}=\left(p_{i}\right)_{i}$, i.e. we compute the "fractal" dimensions of the set

$$
B_{r}(\mathbf{p})=\left\{n \in \mathbb{N}|| \frac{\left|\left\{0 \leq k \leq \gamma(n) \mid d_{k}(n)=i\right\}\right|}{\gamma(n)+1}-p_{i} \mid \leq r \text { for all } i\right\}
$$

in the limit as $r \searrow 0$; and, for a given function $f:\{0,1, \ldots, N-1\} \rightarrow \mathbb{R}$, we compute the "fractal" dimensions of the set of positive integers $n$ such that the sum of $f$ over the digits in $n$ equals a given real number $t$, i.e. we compute the "fractal" dimensions of the set

$$
E_{r}(t)=\left\{n \in \mathbb{N}|| \frac{f\left(d_{0}(n)\right)+f\left(d_{1}(n)\right)+\ldots+f\left(d_{\gamma(n)}(n)\right)}{\gamma(n)+1}-t \mid \leq r\right\}
$$

in the limit as $r \searrow 0$. For example, if $x \approx 6.032078568$ denotes the unique

2000 Mathematics Subject Classification: Primary 11A63; Secondary 11K16, 37D30, $37 \mathrm{~A} 45$.

Key words and phrases: distribution of digits, distribution of sum of digits, Besicovitch-Eggleston sets, fractals subsets of the integers, fractional dimensions of discrete sets, zeta functions. 
real number such that

$$
\sum_{i=0}^{9}(\sin (i)-7 / 10) x^{\sin (i)}=0,
$$

and we let

$$
d=\log \left(\sum_{i=0}^{9} x^{\sin (i)} / x^{7 / 10}\right) / \log (10) \approx 0.8386016690,
$$

then it follows from Corollary 5 that there are roughly $n^{d}$ positive integers $m$ less than $n$ such that the average of the sum of the sines of the decimal digits in $m$ equals $7 / 10$; more precisely, if

$$
a(n)=\frac{\sin \left(d_{0}(n)\right)+\sin \left(d_{1}(n)\right)+\ldots+\sin \left(d_{\gamma(n)}(n)\right)}{\gamma(n)+1}
$$

denotes the average of the sum of the sines of the decimal digits in $n$ and we write

$$
E_{r}=\{n \in \mathbb{N}|| a(n)-7 / 10 \mid \leq r\}, \quad N_{n}\left(E_{r}\right)=\left|\{1, \ldots, n\} \cap E_{r}\right|,
$$

then

$$
\lim _{r \searrow 0} \liminf _{n} \frac{\log N_{n}\left(E_{r}\right)}{\log n}=\lim _{r \searrow 0} \limsup _{n} \frac{\log N_{n}\left(E_{r}\right)}{\log n}=d .
$$

Also, if we define the zeta function $\zeta_{E_{r}}$ of the set $E_{r}$ by

$$
\zeta_{E_{r}}(t)=\sum_{n \in E_{r}} \frac{1}{n^{t}},
$$

and denote the abscissa of convergence of $\zeta_{r}$ by $\sigma_{\mathrm{c}, E_{r}}$, then

$$
\lim _{r \searrow 0} \sigma_{\mathrm{c}, E_{r}}=d .
$$

In Section 1.1 we introduce several natural fractional dimensions and zeta functions of subsets of $\mathbb{N}$. The key idea is that the fractional dimensions and the abscissa of convergence provide natural discrete analogues of the usual fractional dimensions in fractal geometry; indeed, this point of view is supported by Corollaries 4 and 5 which provide natural discrete counterparts of some well-known results in fractal geometry.

In Section 1.2 we define the notion of a continuous deformation of the digits of a positive integer. Using this notion we introduce a very general class of subsets of $\mathbb{N}$ defined in terms of the asymptotic behaviour of the digits in the $N$-ary expansion of the integers. These sets include, for example, the sets in (1.2) and (1.3), i.e. the set of positive integers $n$ such that each digit $i$ of $n$ appears with frequence $p_{i}$ for some probability vector $\mathbf{p}=\left(p_{i}\right)_{i}$, i.e. the set

$$
B_{r}(\mathbf{p})=\left\{n \in \mathbb{N}|| \frac{\left|\left\{0 \leq k \leq \gamma(n) \mid d_{k}(n)=i\right\}\right|}{\gamma(n)+1}-p_{i} \mid \leq r \text { for all } i\right\},
$$


and the set of positive integers $n$ such that the sum of the function $f$ over the digits of $n$ equals a given real number $t$, i.e. the set

$$
E_{r}(t)=\left\{n \in \mathbb{N}|| \frac{f\left(d_{0}(n)\right)+f\left(d_{1}(n)\right)+\ldots+f\left(d_{\gamma(n)}(n)\right)}{\gamma(n)+1}-t \mid \leq r\right\} .
$$

Section 1.3 contains the main result of the paper, viz. Theorem 2. In Theorem 2 we compute the fractional dimensions and abscissa of convergence of the zeta functions of the sets introduced in Section 1.2. The fractional dimensions and abscissa of convergence of the zeta functions will be expressed in terms of entropies of certain probability measures. This provides an interesting connection between dynamical systems and arithmetic functions in number theory.

In Sections 1.4 and 1.5 we apply Theorem 2 to compute the fractional dimensions of the set $B_{r}(\mathbf{p})$ and the set $E_{r}(t)$ (in the limit as $r \searrow 0$ ) (cf. Corollaries 4 and 5). Corollary 4, giving the fractional dimensions of the set $B_{r}(\mathbf{p})$, provides a natural discrete analogue of a classical result due to Besicovitch and Eggleston on the Hausdorff dimension of certain sets of non-normal numbers, and Corollary 5, giving the fractional dimensions of the set $E_{r}(t)$, provides a natural discrete analogue of a result in dynamical systems on the Hausdorff dimension of sets with prescribed ergodic averages.

Finally, in Section 1.6 we consider a more general class of zeta functions associated with subsets of $\mathbb{N}$ and give an upper bound for the abscissa of convergence.

1.1. Fractional dimensions and zeta functions of subsets of $\mathbb{N}$. We will now define various notions of fractional dimensions of a subset of the positive integers $\mathbb{N}$. For $E \subseteq \mathbb{N}$, let $N_{n}(E)=|\{1, \ldots, n\} \cap E|$. Following $[\mathrm{BeF}]$, we define the lower and upper fractal dimension of $E$ by

$$
\underline{\operatorname{dim}}(E)=\liminf _{n} \frac{\log N_{n}(E)}{\log n}, \quad \overline{\operatorname{dim}}(E)=\limsup _{n} \frac{\log N_{n}(E)}{\log n} .
$$

Definitions of dimensions for discrete sets also appear in [BlK, BaT1, BaT2, $\mathrm{Fu}, \mathrm{Na}$. However, these definitions have been designed for other purposes and generally take different values than $\underline{\operatorname{dim}}(E)$ and $\overline{\operatorname{dim}}(E)$. Motivated by the so-called exponent of convergence for limit sets of Kleinian groups, we define the exponent of convergence $\delta(E)$ of $E \subseteq \mathbb{N}$ by

$$
\delta(E)=\sup \left\{t \geq 0 \mid \sum_{n \in E} \frac{1}{n^{t}}=\infty\right\} .
$$

The number $\delta(E)$ can clearly also be defined in terms of Dirichlet series and the corresponding zeta functions. For $E \subseteq \mathbb{N}$ we define the zeta function $\zeta_{E}$ 
of $E$ by

$$
\zeta_{E}(t)=\sum_{n \in E} \frac{1}{n^{t}}
$$

If we let $\sigma_{\mathrm{c}, E}$ and $\sigma_{\mathrm{a}, E}$ denote the abscissa of convergence of $\zeta_{E}$ and the abscissa of absolute convergence of $\zeta_{E}$, then clearly $\sigma_{\mathrm{c}, E}=\sigma_{\mathrm{a}, E}=\delta(E)$. Since the coefficients in the Dirichlet series (1.6) are positive, it follows from the general theory of Dirichlet series (cf. [Ap, Theorems 8.2 and 8.3]) that $\sigma_{\mathrm{c}, E}=\sigma_{\mathrm{a}, E}=\overline{\operatorname{dim}}(E)$. Hence we have

\section{Proposition 1. Let $E$ be a subset of $\mathbb{N}$. Then}

$$
\underline{\operatorname{dim}}(E) \leq \overline{\operatorname{dim}}(E)=\sigma_{\mathrm{c}, E}=\sigma_{\mathrm{a}, E}=\delta(E) \leq 1
$$

The reader is referred to the recent monograph by Lapidus \& van Frankenhuysen $[\mathrm{LvF}]$ for a different attempt to develop a theory of fractal dimensions (of so-called fractal strings) based on zeta functions.

1.2. Subsets of $\mathbb{N}$ defined by continuous deformations digits. We will now introduce the notion of a continuous deformation of the digits of a positive integer.

We first introduce some notation. Let

$$
\Sigma^{*}=\bigcup_{n}\{0,1, \ldots, N-1\}^{n}, \quad \Sigma=\{0,1, \ldots, N-1\}^{\mathbb{N}},
$$

i.e. $\Sigma^{*}$ is the set of all finite strings $\omega_{1} \ldots \omega_{n}$ with elements $\omega_{i}$ from $\{0,1, \ldots$ $\ldots, N-1\}$, and $\Sigma$ is the set of all infinite strings $\omega_{1} \omega_{2} \ldots$ with elements $\omega_{i}$ from $\{0,1, \ldots, N-1\}$. We let $S: \Sigma \rightarrow \Sigma$ denote the shift map, i.e.

$$
S\left(\omega_{1} \omega_{2} \ldots\right)=\omega_{2} \omega_{3} \ldots
$$

For $\omega=\omega_{1} \ldots \omega_{n}, \sigma=\sigma_{1} \ldots \sigma_{m} \in \Sigma^{*}$, we let $\omega \sigma=\omega_{1} \ldots \omega_{n} \sigma_{1} \ldots \sigma_{m} \in \Sigma^{*}$ denote the concatenation of $\omega$ and $\sigma$, and for $\omega \in \Sigma^{*}$, we write

$$
\bar{\omega}=\omega \omega \ldots \in \Sigma \text {. }
$$

Let $\mathcal{P}(\Sigma)$ denote the family of probability measures on $\Sigma$, and let $\mathcal{P}_{\mathrm{S}}(\Sigma)$ denote the family of shift invariant probability measures on $\Sigma$.

Recall that if $n$ is a positive integer, then

$$
n=d_{0}(n)+d_{1}(n) N+d_{2}(n) N^{2}+\ldots+d_{\gamma(n)}(n) N^{\gamma(n)},
$$

where $d_{i}(n) \in\{0,1,2, \ldots, N-1\}$ and $d_{\gamma(n)}(n) \neq 0$, denotes the $N$-ary expansion of $n$. We denote the string $d_{0}(n) d_{1}(n) \ldots d_{\gamma(n)}(n) \in \Sigma^{*}$ of $N$-ary digits of $n$ by $d(n)$, i.e.

$$
d(n):=d_{0}(n) d_{1}(n) \ldots d_{\gamma(n)}(n) \in \Sigma^{*} .
$$


(We emphasize that $d(n)$ is not the product of the $N$-ary digits in $n$. If, for example, $N=7$ and $n=2+5 \cdot 7+4 \cdot 7^{2}+6 \cdot 7^{4}$, then $d(n)$ equals the string 25406.)

With each positive integer $n$ we will now associate a natural probability measure $I(n)$ on $\Sigma$. Define $I: \mathbb{N} \rightarrow \mathcal{P}(\Sigma)$ by

$$
I(n)=\frac{1}{\gamma(n)+1} \sum_{k=0}^{\gamma(n)} \delta_{S^{k}}(\overline{d(n)})
$$

where $\delta_{x}$ denotes the Dirac measure concentrated at $x$. The probability measure $I(n)$ describes in a measure-theoretic way the distribution of the digits in $n$, and enables us to use methods from ergodic theory and large deviations. By a continuous deformation of the digits of a positive integer, we mean a pair $(X, \Xi)$ where $X$ is a metric space and $\Xi: \mathcal{P}(\Sigma) \rightarrow X$ is continuous with respect to the weak topology; we will think of the composite $n \mapsto \Xi(I(n))$ as a continuous deformation of the digits of $n$. Let $(X, \Xi)$ be a continuous deformation. For a subset $C$ of $X$ and $r>0$, we let

$$
\Delta_{r}(C)=\{n \in \mathbb{N} \mid \operatorname{dist}(\Xi(I(n)), C) \leq r\} .
$$

If $C=\{x\}$ for $x \in X$, we will write $\Delta_{r}(x)=\Delta_{r}(\{x\})$. For different choices of $X$ and $\Xi$ we obtain sets describing the distribution of different numbertheoretic functions. We emphasize that we do not assume that $X$ is a vector space and that the deformation map $\Xi: \mathcal{P}(\Sigma) \rightarrow X$ is affine. Indeed, interesting examples are obtained for certain non-affine deformations; cf., for example, the non-affine deformation in example (1.11) below. For a digit $i \in\{0,1, \ldots, N-1\}$ we define the cylinder $[i]$ generated by $i$ by

$$
[i]=\left\{\omega_{1} \omega_{2} \ldots \in \Sigma \mid \omega_{1}=i\right\} .
$$

- Distribution of digits: Let $X=\mathbb{R}^{N}$ and define $\Xi: \mathcal{P}(\Sigma) \rightarrow X$ by

$$
\Xi(\mu)=(\mu([i]))_{i}
$$

Then

$$
\Delta_{r}(C)=\left\{n \in \mathbb{N} \mid \operatorname{dist}\left(\left(\frac{\left|\left\{0 \leq k \leq \gamma(n) \mid d_{k}(n)=i\right\}\right|}{\gamma(n)+1}\right)_{i}, C\right) \leq r\right\} .
$$

- Distribution of sum of digits: Let $X=\mathbb{R}$ and define $\Xi: \mathcal{P}(\Sigma) \rightarrow X$ by

$$
\Xi(\mu)=\sum_{i=0,1, \ldots, N-1} i \mu([i]) \text {. }
$$

Then

$$
\Delta_{r}(C)=\left\{n \in \mathbb{N} \mid \operatorname{dist}\left(\frac{d_{0}(n)+d_{1}(n)+\ldots+d_{\gamma(n)}(n)}{\gamma(n)+1}, C\right) \leq r\right\} .
$$

- Distribution of powers of sum of functions of digits: Fix a positive integer $m$ and let $f:\{0,1, \ldots, N-1\} \rightarrow \mathbb{R}$ be a function. Let $X=\mathbb{R}$ and 
define $\Xi: \mathcal{P}(\Sigma) \rightarrow X$ by

$$
\Xi(\mu)=\sum_{i_{1}, \ldots, i_{m}=0,1, \ldots, N-1} f\left(i_{1}\right) \ldots f\left(i_{m}\right) \mu\left(\left[i_{1}\right]\right) \ldots \mu\left(\left[i_{m}\right]\right) .
$$

Then

$$
\Delta_{r}(C)=\left\{n \in \mathbb{N} \mid \operatorname{dist}\left(\left(\frac{f\left(d_{0}(n)\right)+\ldots+f\left(d_{\gamma(n)}(n)\right)}{\gamma(n)+1}\right)^{m}, C\right) \leq r\right\} .
$$

1.3. Dimensions of subsets of $\mathbb{N}$ defined by continuous deformations of digits. Theorem 2 below computes the fractional dimensions of $\Delta_{r}(C)$ (in the limit as $r \searrow 0)$. For a continuous deformation $(X, \Xi)$ and $x \in X$, we define $H(x)$ by

$$
H(x)=\sup _{\substack{\mu \in \mathcal{P}_{\mathrm{S}}(\Sigma) \\ \Xi(\mu)=x}} \frac{h(\mu)}{\log N}
$$

where $h(\mu)$ denotes the entropy of $\mu$ (as usual, we put $\sup _{\mu \in \emptyset} \frac{h(\mu)}{\log N}=0$ ).

Theorem 2. Let $X$ be a metric space and let $\Xi: \mathcal{P}(\Sigma) \rightarrow X$ be continuous with respect to the weak topology.

(i) If $C \nsubseteq \Xi(\mathcal{P}(\Sigma))$, then

$$
\lim _{r \searrow 0} \underline{\operatorname{dim}}\left(\Delta_{r}(C)\right)=\lim _{r \searrow 0} \overline{\operatorname{dim}}\left(\Delta_{r}(C)\right)=\lim _{r \searrow 0} \delta\left(\Delta_{r}(C)\right)=0 .
$$

(ii) If $C \subseteq \Xi(\mathcal{P}(\Sigma))$ is closed, then

$$
\lim _{r \searrow 0} \underline{\operatorname{dim}}\left(\Delta_{r}(C)\right)=\lim _{r \searrow 0} \overline{\operatorname{dim}}\left(\Delta_{r}(C)\right)=\lim _{r \searrow 0} \delta\left(\Delta_{r}(C)\right)=\sup _{x \in C} H(x) .
$$

In particular, for $x \in \Xi(\mathcal{P}(\Sigma))$ we have

$$
\lim _{r \searrow 0} \underline{\operatorname{dim}}\left(\Delta_{r}(x)\right)=\lim _{r \searrow 0} \overline{\operatorname{dim}}\left(\Delta_{r}(x)\right)=\lim _{r \searrow 0} \delta\left(\Delta_{r}(x)\right)=H(x) .
$$

To prove Theorem 2, it clearly suffices to prove the upper bound

$$
\limsup _{r \searrow 0} \delta\left(\Delta_{r}(C)\right) \leq \sup _{x \in C} H(x)
$$

and the lower bound

$$
\liminf _{r \searrow 0} \underline{\operatorname{dim}}\left(\Delta_{r}(C)\right) \geq \sup _{x \in C} H(x) .
$$

The upper bound (1.13) follows from a more general result, Theorem 6, in Section 1.6. Theorem 6 is proved in Section 3 using methods from large deviation theory. The lower bound (1.14) is proved in Section 4 using arguments from ergodic theory.

With the use of Proposition 1, Theorem 2 can clearly also be formulated in terms of abscissa of convergence of Dirichlet series. 
Theorem 3. Let $X$ be a metric space and let $\Xi: \mathcal{P}(\Sigma) \rightarrow X$ be continuous with respect to the weak topology. Let $C$ be a subset of $X$, and let $\sigma_{\mathrm{c}, r}$ and $\sigma_{\mathrm{a}, r}$ denote the abscissa of convergence and the abscissa of absolute convergence of the Dirichlet series

$$
\zeta_{\Delta_{r}(C)}(t)=\sum_{n \in \Delta_{r}(C)} \frac{1}{n^{t}} .
$$

(i) If $C \nsubseteq \Xi(\mathcal{P}(\Sigma))$, then

$$
\lim _{r \searrow 0} \sigma_{\mathrm{c}, r}=\lim _{r \searrow 0} \sigma_{\mathrm{a}, r}=0 .
$$

(ii) If $C \subseteq \Xi(\mathcal{P}(\Sigma))$ is closed, then

$$
\lim _{r \searrow 0} \sigma_{\mathrm{c}, r}=\lim _{r \searrow 0} \sigma_{\mathrm{a}, r}=\sup _{x \in C} H(x) .
$$

For particular choices of $X$ and $\Xi$, the supremum

$$
H(x)=\sup _{\substack{\mu \in \mathcal{P}_{\mathrm{S}}(\Sigma) \\ \Xi(\mu)=x}} \frac{h(\mu)}{\log N}
$$

can be computed explicitly. Below we consider two interesting examples.

1.4. An application: Besicovitch-Eggleston subsets of $\mathbb{N}$. The first application of Theorem 2 investigates the frequency of the digits in the $N$-ary expansion of integers, and provides a natural discrete analogue of a classical result due to Besicovitch and Eggleston.

Corollary 4. Let $X=\mathbb{R}^{N}$ and define $\Xi: \mathcal{P}(\Sigma) \rightarrow X$ by $\Xi(\mu)=$ $(\mu([i]))_{i}$. For $C \subseteq \mathbb{R}^{N}$, we have

$\Delta_{r}(C)=B_{r}(C):=\left\{n \in \mathbb{N} \mid \operatorname{dist}\left(\left(\frac{\left|\left\{0 \leq k \leq \gamma(n) \mid d_{k}(n)=i\right\}\right|}{\gamma(n)+1}\right)_{i}, C\right) \leq r\right\}$.

Let $\Delta=\left\{\left(p_{0}, p_{1}, \ldots, p_{N-1}\right) \mid p_{i} \geq 0, \sum_{i} p_{i}=1\right\}$ denote the set of $N$ dimensional probability vectors. Then $\Xi\left(\mathcal{P}_{\mathrm{S}}(\Sigma)\right)=\Delta$ and

$$
H(\mathbf{p})=-\frac{\sum_{i} p_{i} \log p_{i}}{\log N} \quad \text { for } \mathbf{p}=\left(p_{0}, p_{1}, \ldots, p_{N-1}\right) \in \Delta .
$$

If $C \subseteq \Delta$ is closed, then

$$
\begin{aligned}
\lim _{r \searrow 0} \underline{\operatorname{dim}}\left(B_{r}(C)\right) & =\lim _{r \searrow 0} \overline{\operatorname{dim}}\left(B_{r}(C)\right)=\lim _{r \searrow 0} \delta\left(B_{r}(C)\right) \\
& =\sup _{\left(p_{0}, p_{1}, \ldots, p_{N-1}\right) \in C}-\frac{\sum_{i} p_{i} \log p_{i}}{\log N} .
\end{aligned}
$$


In particular, for $\mathbf{p}=\left(p_{0}, p_{1}, \ldots, p_{N-1}\right) \in \Delta$ we have (where we have written $\left.B_{r}(\mathbf{p})=B_{r}(\{\mathbf{p}\})\right)$

$\lim _{r \searrow 0} \underline{\operatorname{dim}}\left(B_{r}(\mathbf{p})\right)=\lim _{r \searrow 0} \overline{\operatorname{dim}}\left(B_{r}(\mathbf{p})\right)=\lim _{r \searrow 0} \delta\left(B_{r}(\mathbf{p})\right)=-\frac{\sum_{i} p_{i} \log p_{i}}{\log N}$.

Proof. Using standard properties of the entropy, it is easily seen that if $\mathbf{p}=\left(p_{0}, p_{1}, \ldots, p_{N-1}\right) \in \Delta$, then

$$
H(\mathbf{p}):=\sup _{\substack{\mu \in \mathcal{P}_{\mathrm{S}}(\Sigma) \\(\mu([i]))_{i}=\mathbf{p}}} \frac{h(\mu)}{\log N}=-\frac{\sum_{i} p_{i} \log p_{i}}{\log N} .
$$

The desired result now follows from Theorem 2.

The result in Corollary 4 was first obtained in [O12] and provides a natural discrete analogue of a classical result due to Besicovitch and Eggleston on the Hausdorff dimension of certain sets of non-normal numbers. For a real number $x \in[0,1]$, we let

$$
x=\sum_{n=1}^{\infty} \frac{\varepsilon_{n}(x)}{N^{n}}
$$

where $\varepsilon_{n}(x) \in\{0,1, \ldots, N-1\}$, denote the unique non-terminating $N$-ary expansion of $x$. Let $B(\mathbf{p})$ denote the set of reals $x$ such that the frequency of the digit $i$ among the first $n$ digits of $x$ approaches $p_{i}$ as $n \rightarrow \infty$ for all $i \in\{0,1, \ldots, N-1\}$, i.e. the set

$$
B(\mathbf{p})=\left\{x \in[0,1] \mid \frac{\left|\left\{1 \leq k \leq n \mid \varepsilon_{k}(x)=i\right\}\right|}{n} \rightarrow p_{i} \text { for all } i\right\} .
$$

Besicovitch and Eggleston proved that

$$
\operatorname{dim} B(\mathbf{p})=-\frac{\sum_{i} p_{i} \log p_{i}}{\log N}
$$

where dim denotes the Hausdorff dimension. Formula (1.16) was first proved by Besicovitch [Be] for $N=2$ in 1934, and later for general $N$ by Eggleston [Eg] in 1949.

Other references to the distribution of digits in integers can be found in [DT2, NS, Sh].

1.5. An application: subsets of $\mathbb{N}$ with prescribed digit sum averages. The second application of Theorem 2 studies the distribution of averages of sums of functions of digits, and provides a natural discrete analogue of some recent results from dynamical systems on the dimension of sets with prescribed ergodic average. We note that there is a vast literature discussing other approaches to the study of the asymptotic properties of the sum of digits (cf. [BS, Bu, Co, De, DT1, DT3, GKPT, K, KC]). 
Corollary 5. Let $f:\{0,1, \ldots, N-1\} \rightarrow \mathbb{R}$ be a function. Let $X=\mathbb{R}$ and define $\Xi: \mathcal{P}(\Sigma) \rightarrow X$ by $\Xi(\mu)=\sum_{i} f(i) \mu([i])$ (recall that the cylinder $[i]$ is defined by $\left.[i]=\left\{\omega_{1} \omega_{2} \ldots \in \Sigma \mid \omega_{1}=i\right\}\right)$. For $C \subseteq \mathbb{R}$, we have

$$
\begin{aligned}
\Delta_{r}(C) & =E_{r}(C) \\
& :=\left\{n \in \mathbb{N} \mid \operatorname{dist}\left(\frac{f\left(d_{0}(n)\right)+f\left(d_{1}(n)\right)+\ldots+f\left(d_{\gamma(n)}(n)\right)}{\gamma(n)+1}, C\right) \leq r\right\} .
\end{aligned}
$$

Let $I=\left[0, \max _{i} f(i)\right]$ and write

$$
T_{t}=\left\{x \geq 0 \mid \sum_{i=0}^{N-1}(f(i)-t) x^{f(i)}=0\right\} \quad \text { for } t \in \mathbb{R} .
$$

Then $\Xi\left(\mathcal{P}_{\mathrm{S}}(\Sigma)\right)=I$ and

$$
H(t):=\sup _{\substack{\mu \in \mathcal{P}_{\mathrm{S}}(\Sigma) \\ \sum_{i} f(i) \mu([i])=t}} \frac{h(\mu)}{\log N}=\max _{x \in T_{t}} \frac{1}{\log N} \log \left(\frac{\sum_{i} x^{f(i)}}{x^{t}}\right) \quad \text { for } t \in I .
$$

If $C \subseteq I$ is closed, then

$$
\begin{aligned}
\lim _{r \searrow 0} \underline{\operatorname{dim}}\left(E_{r}(C)\right) & =\lim _{r \searrow 0} \overline{\operatorname{dim}}\left(E_{r}(C)\right)=\lim _{r \searrow 0} \delta\left(E_{r}(C)\right) \\
& =\sup _{t \in C} \max _{x \in T_{t}} \frac{1}{\log N} \log \left(\frac{\sum_{i} x^{f(i)}}{x^{t}}\right) .
\end{aligned}
$$

In particular, for $t \in I$ we have (where we have written $E_{r}(t)=E_{r}(\{t\})$ )

$$
\begin{aligned}
\lim _{r \searrow 0} \underline{\operatorname{dim}}\left(E_{r}(t)\right) & =\lim _{r \searrow 0} \overline{\operatorname{dim}}\left(E_{r}(t)\right)=\lim _{r \searrow 0} \delta\left(E_{r}(t)\right) \\
& =\max _{x \in T_{t}} \frac{1}{\log N} \log \left(\frac{\sum_{i} x^{f(i)}}{x^{t}}\right) .
\end{aligned}
$$

Proof. We must prove that if $t \in I$, then

$$
H(t):=\sup _{\substack{\mu \in \mathcal{P}_{\mathrm{S}}(\Sigma) \\ \sum_{i} f(i) \mu([i])=t}} \frac{h(\mu)}{\log N}=\max _{x \in T_{t}} \frac{1}{\log N} \log \left(\frac{\sum_{i} x^{f(i)}}{x^{t}}\right) .
$$

Let $M$ denote the right hand side of (1.17).

We first prove that $H(t) \geq M$. Fix $x \in T_{t}$ and write $p_{i}=x^{f(i)} / \sum_{j} x^{f(j)}$. Then $\left(p_{i}\right)_{i}$ is a probability vector and we can define a shift invariant probability measure $\nu$ on $\Sigma$ by $\nu=\mathrm{X}_{n=1}^{\infty}\left(\sum_{i} p_{i} \delta_{i}\right)$. Clearly $\sum_{i} f(i) \nu([i])=$ $\sum_{i} f(i) p_{i}=t$, whence

$$
H(t) \geq \frac{h(\nu)}{\log N}=-\frac{\sum_{i} p_{i} \log p_{i}}{\log N}=\frac{1}{\log N} \log \left(\frac{\sum_{i} x^{f(i)}}{x^{t}}\right) .
$$

Taking maximum over all $x \in T_{t}$ shows that $M \leq H(t)$. 
Next we prove that $H(t) \leq M$. Let $\Delta=\left\{\left(p_{0}, p_{1}, \ldots, p_{N-1}\right) \mid p_{i} \geq 0\right.$, $\left.\sum_{i} p_{i}=1\right\}$ denote the set of $N$-dimensional probability vectors. Let $\mu \in$ $\mathcal{P}_{\mathrm{S}}(\Sigma)$ with $\sum_{i} f(i) \mu([i])=t$. Then clearly

$$
H(t) \leq-\frac{\sum_{i} \mu([i]) \log \mu([i])}{\log N} \leq \sup _{\substack{\left(p_{0}, p_{1}, \ldots, p_{N-1}\right) \in \Delta \\ \sum_{i} f(i) p_{i}=t}}-\frac{\sum_{i} p_{i} \log p_{i}}{\log N}
$$

Finally, a standard calculus argument using Lagrange multipliers shows that

$$
\sup _{\substack{\left(p_{0}, p_{1}, \ldots, p_{N-1}\right) \in \Delta \\ \sum_{i} f(i) p_{i}=t}}-\frac{\sum_{i} p_{i} \log p_{i}}{\log N} \leq M
$$

This shows that $H(t) \leq M$.

The desired result now follows from (1.17) and Theorem 2.

The result in Corollary 5, i.e. the equality

$$
\begin{aligned}
\lim _{r \searrow 0} \underline{\operatorname{dim}}\left(\Delta_{r}(t)\right)=\lim _{r \searrow 0} \overline{\operatorname{dim}}\left(\Delta_{r}(t)\right) & =\sup _{\substack{\mu \in \mathcal{P}_{\mathrm{S}}(\Sigma) \\
\sum_{i} f(i) \mu([i])=t}} \frac{h(\mu)}{\log N} \\
( & \left.=\max _{x \in T_{t}} \frac{1}{\log N} \log \left(\frac{\sum_{i} x^{f(i)}}{x^{t}}\right)\right),
\end{aligned}
$$

provides a natural discrete analogue of a result from dynamical systems on the dimension of sets with prescribed ergodic average. Let $f: \Sigma \rightarrow \mathbb{R}$ be a continuous function and $t \in \mathbb{R}$. Let $E(t)$ denote the set of $\omega \in \Sigma$ such that the ergodic average of $f$ over the orbit $\left(\omega, S \omega, S^{2} \omega, S^{3} \omega, \ldots\right)$ of $\omega$ equals $t$, i.e.

$$
E(t)=\left\{\omega \in \Sigma \mid \frac{f(\omega)+f(S(\omega))+\ldots+f\left(S^{n-1}(\omega)\right)}{n} \rightarrow t\right\} .
$$

Then

$$
\operatorname{dim} E(t)=\sup _{\substack{\mu \in \mathcal{P}_{\mathrm{S}}(\Sigma) \\ \int f d \mu=t}} \frac{h(\mu)}{\log N}
$$

This result was proved for Hölder continuous functions $f$ by Pesin \& Weiss $[\mathrm{PW}]$ and has recently been extended to arbitrary continuous functions by Fan et al. [FFW] and Olsen [Ol1].

1.6. Some generalizations. In this section we introduce a more general class of zeta functions than those introduced in Section 1.1, and we study the abscissa of convergence and Euler products of these.

Let $\mathbf{s}=\left(s_{0}, s_{1}, \ldots, s_{N-1}\right)$ be a family of real numbers with $s_{i} \in(0,1)$. Recall that $\Sigma^{*}=\bigcup_{n}\{0,1, \ldots, N-1\}^{n}$ denotes the set of all finite strings $\omega_{1} \ldots \omega_{n}$ with elements $\omega_{i}$ from $\{0,1, \ldots, N-1\}$. For a finite string $\omega=$ 
$\omega_{1} \ldots \omega_{n} \in \Sigma^{*}$ with elements $\omega_{i}$ from $\{0,1, \ldots, N-1\}$, we write

$$
s_{\omega}=s_{\omega_{1}} \ldots s_{\omega_{n}} .
$$

Also, recall that if $n \in \mathbb{N}$ is a positive integer, then

$$
d(n)=d_{0}(n) d_{1}(n) \ldots d_{\gamma(n)}(n) \in \Sigma^{*}
$$

denotes the finite string of $N$-ary digits $d_{0}(n), d_{1}(n), \ldots, d_{\gamma(n)}(n)$ of $n$. In particular, we have $s_{d(n)}=s_{d_{0}(n)} s_{d_{1}(n)} \ldots s_{d_{\gamma(n)}(n)}$. For a subset $E$ of $\mathbb{N}$, we define the s-exponent of convergence $\delta(E ; \mathbf{s})$ of $E$ by

$$
\delta(E ; \mathbf{s})=\sup \left\{t \geq 0 \mid \sum_{n \in E} s_{d(n)}^{t}=\infty\right\} .
$$

As in Section 1.1, the exponent $\delta(E ; \mathbf{s})$ can also be defined in terms of zeta functions. We define the $\mathbf{s}$ zeta function of $E \subseteq \mathbb{N}$ by

$$
\zeta_{E}(t ; \mathbf{s})=\sum_{n \in E} s_{d(n)}^{t} .
$$

If we let $\sigma_{\mathrm{c}, E}(\mathbf{s})$ denote abscissa of convergence of $\zeta_{E}(t ; \mathbf{s})$, then clearly $\sigma_{\mathrm{c}, E}(\mathbf{s})=\delta(E ; \mathbf{s})$. In the theorem below we give an upper bound for $\sigma_{\mathrm{c}, \Delta_{r}(C)}(\mathbf{s})=\delta\left(\Delta_{r}(C) ; \mathbf{s}\right)$; recall that the set $\Delta_{r}(C)$ is defined in (1.8).

THEOREM 6. Let $\mathbf{s}=\left(s_{0}, s_{1}, \ldots, s_{N-1}\right)$ be a family of real numbers with $s_{i} \in(0,1)$. Let $X$ be a metric space and let $\Xi: \mathcal{P}(\Sigma) \rightarrow X$ be continuous with respect to the weak topology.

(i) If $C \nsubseteq \Xi(\mathcal{P}(\Sigma))$, then

$$
\lim _{r \searrow 0} \delta\left(\Delta_{r}(C) ; \mathbf{s}\right)=0 .
$$

(ii) If $C \subseteq \Xi(\mathcal{P}(\Sigma))$ is closed, then

$$
\limsup _{r \searrow 0} \delta\left(\Delta_{r}(C) ; \mathbf{s}\right) \leq \sup _{\substack{\mu \in \mathcal{P}_{\mathrm{S}}(\Sigma) \\ \Xi(\mu) \in C}}-\frac{h(\mu)}{\sum_{i} \mu([i]) \log s_{i}} .
$$

We will now prove an Euler product for $\zeta_{\Delta_{r}(C)}(t ; \mathbf{s})$. We define the modified $\mathbf{s}$ zeta function of a subset $E$ of $\mathbb{N}$ by

$$
Z_{E}(t ; \mathbf{s})=\sum_{n \in E} \frac{1}{\gamma(n)+1} s_{d(n)}^{t} .
$$

Next we define the Euler product of $E$. A finite string $\omega \in \Sigma^{*}$ is called periodic if there exists a finite string $\sigma \in \Sigma^{*}$ and a positive integer $n \geq 2$ such that $\omega=\sigma \ldots \sigma$ where $n$ copies of $\sigma$ have been concatenated. A string is called primitive if it is not periodic. We define the set $\mathbb{P}$ of $N$-ary primitive integers by

$$
\mathbb{P}=\{n \in \mathbb{N} \mid d(n) \text { is primitive }\}
$$


The $\mathbf{s}$ Euler product of $E$ is now defined by

$$
Q_{E}(t ; \mathbf{s})=\prod_{n \in E \cap \mathbb{P}}\left(\frac{1}{1-s_{d(n)}^{t}}\right)^{1 /(\gamma(n)+1)} .
$$

THEOREM 7. Let $\mathbf{s}=\left(s_{0}, s_{1}, \ldots, s_{N-1}\right)$ be a family of real numbers with $s_{i} \in(0,1)$. Let $X$ be a metric space and let $\Xi: \mathcal{P}(\Sigma) \rightarrow X$ be continuous with respect to the weak topology. Let $C \subseteq X$ be a closed subset of $X$ and $r>0$.

(i) For $t \in \mathbb{C}$ with $\operatorname{Re} t>\sigma_{\mathrm{c}, \Delta_{r}(C)}(\mathbf{s})$ we have

$$
\exp Z_{\Delta_{r}(C)}(t ; \mathbf{s})=Q_{\Delta_{r}(C)}(t ; \mathbf{s}) .
$$

(ii) Assume that $\mathbf{s}=(s, \ldots, s)$ for some $s \in(0,1)$. For $t \in \mathbb{C}$ with $\operatorname{Re} t>\sigma_{\mathrm{c}, \Delta_{r}(C)}(\mathbf{s})$ we have

$$
\frac{d}{d t} Z_{\Delta_{r}(C)}(t ; \mathbf{s})=\zeta_{\Delta_{r}(C)}(t ; \mathbf{s}) \log s .
$$

In particular, for $t \in \mathbb{C}$ with $\operatorname{Re} t>\sigma_{\mathrm{c}, \Delta_{r}(C)}(\mathbf{s})$ we have

$$
\zeta_{\Delta_{r}(C)}(t ; \mathbf{s}) \log s=\frac{d}{d t} \log Q_{\Delta_{r}(C)}(t ; \mathbf{s}) .
$$

In Section 2 we collect some notation. In Section 3 we prove Theorem 6 and the upper bound (1.13), and in Section 4 we prove the lower bound (1.14). Finally, in Section 5 we prove Theorem 7.

2. Notation and preliminary results. In this section we collect some notation. Let

$$
\Sigma^{n}=\left\{\omega_{1} \ldots \omega_{n} \mid \omega_{i}=0,1, \ldots, N-1\right\}
$$

and recall that

$$
\Sigma^{*}=\bigcup_{n} \Sigma^{n}, \quad \Sigma=\left\{\omega_{1} \omega_{2} \ldots \mid \omega_{i}=0,1, \ldots, N-1\right\},
$$

i.e. $\Sigma^{n}$ is the family of all strings of length $n, \Sigma^{*}$ is the family of all finite strings, and $\Sigma$ is the family of all infinite strings. For $\omega \in \Sigma^{n}$, we will write $|\omega|=n$. For $\omega=\omega_{1} \ldots \omega_{n} \in \Sigma^{n}$ and a positive integer $m$ with $m \leq n$, or for $\omega=\omega_{1} \omega_{2} \ldots \in \Sigma$ and a positive integer $m$, let $\omega \mid m=\omega_{1} \ldots \omega_{m}$ denote the truncation of $\omega$ to the $m$ th place. The cylinder $[\omega]$ generated by a finite string $\omega \in \Sigma^{n}$ is defined by

$$
[\omega]=\{\sigma \in \Sigma|\sigma| n=\omega\} .
$$

3. Proof of Theorem 6 and the upper bound (1.13). To prove Theorem 6 we need some results from large deviation theory. In particular, we need Varadhan's [Va] large deviation theorem (Theorem 3.1(i) below), 
and a non-trivial application of this (viz. Theorem 3.1(ii)) providing first order asymptotics of certain "Boltzmann distributions".

Definition. Let $X$ be a complete separable metric space and let $\left(P_{n}\right)_{n}$ be a sequence of probability measures on $X$. Let $\left(a_{n}\right)_{n}$ be a sequence of positive numbers with $a_{n} \rightarrow \infty$ and let $I: X \rightarrow[0, \infty]$ be a lower semicontinuous function with compact level sets. The sequence $\left(P_{n}\right)_{n}$ is said to have the large deviation property with constants $\left(a_{n}\right)_{n}$ and rate function $I$ if the following hold:

(i) for each closed subset $K$ of $X$ we have

$$
\limsup _{n} \frac{1}{a_{n}} \log P_{n}(K) \leq-\inf _{x \in K} I(x) ;
$$

(ii) for each open subset $G$ of $X$ we have

$$
\liminf _{n} \frac{1}{a_{n}} \log P_{n}(G) \geq-\inf _{x \in G} I(x) .
$$

TheOREM 3.1. Let $X$ be a complete separable metric space and let $\left(P_{n}\right)_{n}$ be a sequence of probability measures on $X$. Assume that the sequence $\left(P_{n}\right)_{n}$ has the large deviation property with constants $\left(a_{n}\right)_{n}$ and rate function $I$. Let $F: X \rightarrow \mathbb{R}$ be a continuous function such that

$$
\int \exp \left(a_{n} F\right) d P_{n}<\infty \quad \text { for all } n,
$$

and

$$
\lim _{M \rightarrow \infty} \limsup _{n} \frac{1}{a_{n}} \log \int_{\{M \leq F\}} \exp \left(a_{n} F\right) d P_{n}=-\infty .
$$

(Observe that the above conditions are satisfied if $F$ is bounded.) Then the following statements hold:

(i) We have

$$
\lim _{n} \frac{1}{a_{n}} \log \int \exp \left(a_{n} F\right) d P_{n}=-\inf _{x \in X}(I(x)-F(x)) .
$$

(ii) For each $n$ define a probability measure $Q_{n}$ on $X$ by

$$
Q_{n}(E)=\frac{\int_{E} \exp \left(a_{n} F\right) d P_{n}}{\int \exp \left(a_{n} F\right) d P_{n}} .
$$

Then the sequence $\left(Q_{n}\right)_{n}$ has the large deviation property with constants $\left(a_{n}\right)_{n}$ and rate function $(I-F)-\inf _{x \in X}(I(x)-F(x))$.

Proof. Statement (i) follows from [El, Theorem II.7.1] or [DZ, Theorem 4.3.1], and statement (ii) follows from [El, Theorem II.7.2].

Lemma 3.2. Let $X$ be a metric space and let $f: X \rightarrow \mathbb{R}$ be an upper semicontinuous function. Let $K_{1}, K_{2}, \ldots \subseteq X$ be non-empty compact subsets 
of $X$ with $K_{1} \supseteq K_{2} \supseteq \ldots$ Then

$$
\inf _{k} \sup _{t \in K_{k}} f(t)=\sup _{t \in \bigcap_{k} K_{k}} f(t) .
$$

Proof. This result follows by a standard compactness argument, and the details are therefore omitted.

Recall that if $\omega \in \Sigma^{*}$, then we define $\bar{\omega} \in \Sigma$ by $\bar{\omega}=\omega \omega \ldots$ We now define $L: \Sigma^{*} \rightarrow \mathcal{P}(\Sigma)$ by

$$
L(\omega)=\frac{1}{n} \sum_{k=1}^{n} \delta_{S^{k} \bar{\omega}} \quad \text { for } \omega \in \Sigma^{n} .
$$

Proposition 3.3. Let $\mathbf{s}=\left(s_{0}, s_{1}, \ldots, s_{N-1}\right)$ be a list of numbers with $s_{i} \in(0,1)$ and define $\Phi: \Sigma \rightarrow \mathbb{R}$ by

$$
\Phi(\omega)=\log s_{\omega_{1}} \quad \text { for } \omega=\omega_{1} \omega_{2} \ldots \in \Sigma .
$$

Write $s_{\omega}=s_{\omega_{1}} \ldots s_{\omega_{n}}$ for $\omega=\omega_{1} \ldots \omega_{n} \in \Sigma^{n}$. Let $X$ be a metric space and let $\Xi: \mathcal{P}(\Sigma) \rightarrow X$ be continuous with respect to the weak topology. Let $C \subseteq X$ be a closed subset of $X$ and $r>0$. If

$$
t>\sup _{\substack{\mu \in \mathcal{P}_{\mathrm{S}}(\Sigma) \\ \operatorname{dist}(\Xi(\mu), C) \leq r}}-\frac{h(\mu)}{\int \Phi d \mu}
$$

then

$$
\sum_{m} \sum_{\substack{\omega \in \Sigma^{m} \\ \operatorname{dist}(\Xi(L(\omega)), C) \leq r}} s_{\omega}^{t}<\infty .
$$

Proof. We first prove that

(3.1) $\quad \limsup _{m} \frac{1}{m} \log \sum_{\substack{\sigma \in \Sigma^{m} \\ \operatorname{dist}(\Xi(L(\sigma)), C) \leq r}} s_{\sigma}^{u \leq} \sup _{\substack{\mu \in \mathcal{P}_{\mathrm{S}}(\Sigma) \\ \operatorname{dist}(\Xi(\mu), C) \leq r}}\left(u \int \Phi d \mu+h(\mu)\right)$

for all $u>0$. Fix $u>0$. For a positive integer $m$ we define $L_{m}: \Sigma \rightarrow$ $\mathcal{P}_{\mathrm{S}}(\Sigma)$ by

$$
L_{m}(\omega)=L(\overline{\omega \mid m})=\frac{1}{m} \sum_{k=0}^{m-1} \delta_{S^{k}} \overline{\omega \mid m} .
$$

Next, let $P$ denote the probability measure on $\Sigma$ defined by

$$
P=\underset{n=1}{\infty}\left(\sum_{i=1}^{N} \frac{1}{N} \delta_{i}\right)
$$

Finally, we define $F: \mathcal{P}(\Sigma) \rightarrow \mathbb{R}$ by

$$
F(\mu)=u \int \Phi d \mu
$$


and for $m \in \mathbb{N}$, we define probability measures $P_{m}, Q_{m} \in \mathcal{P}\left(\mathcal{P}_{\mathrm{S}}(\Sigma)\right)$ by

$$
P_{m}=P \circ L_{m}^{-1}, \quad Q_{m}(E)=\frac{\int_{E} \exp (m F) d P_{m}}{\int \exp (m F) d P_{m}} \quad \text { for } E \subseteq \mathcal{P}_{\mathrm{S}}(\Sigma) .
$$

It now follows that

$$
\begin{aligned}
& \int_{\left\{\tau \in \Sigma \mid \operatorname{dist}\left(\Xi\left(L_{m}(\tau)\right), C\right) \leq r\right\}} s_{\omega \mid m}^{u} d P(\omega) \\
& =\sum_{\sigma \in \Sigma^{m}} \int_{[\sigma] \cap\left\{\tau \in \Sigma \mid \operatorname{dist}\left(\Xi\left(L_{m}(\tau)\right), C\right) \leq r\right\}} s_{\omega \mid m}^{u} d P(\omega) \\
& =\sum_{\sigma \in \Sigma^{m}} s_{\sigma}^{t} P\left([\sigma] \cap\left\{\tau \in \Sigma \mid \operatorname{dist}\left(\Xi\left(L_{m}(\tau)\right), C\right) \leq r\right\}\right) \\
& \geq \sum_{\substack{\sigma \in \Sigma^{m} \\
\operatorname{dist}(\Xi(L(\sigma)), C) \leq r}} s_{\sigma}^{u} P([\sigma])=\sum_{\substack{\sigma \in \Sigma^{m} \\
\operatorname{dist}(\Xi(L(\sigma)), C) \leq r}} s_{\sigma}^{u} N^{-m} .
\end{aligned}
$$

Hence

$$
\begin{aligned}
& \sum_{\substack{\sigma \in \Sigma^{m} \\
\operatorname{dist}(\Xi(L(\sigma)), C) \leq r}} s_{\sigma}^{u} \int_{\left\{\tau \in \Sigma \mid \operatorname{dist}\left(\Xi\left(L_{m}(\tau)\right), C\right) \leq r\right\}} s_{\omega \mid m}^{u} d P(\omega) \\
& \leq N^{m} \int_{\left\{\tau \in \Sigma \mid \operatorname{dist}\left(\Xi\left(L_{m}(\tau)\right), C\right) \leq r\right\}} \exp \left(u \sum_{k=0}^{m-1} \Phi S^{k} \omega\right) d P(\omega) \\
& =N^{m} \int_{\left\{\tau \in \Sigma \mid \operatorname{dist}\left(\Xi\left(L_{m}(\tau)\right), C\right) \leq r\right\}} \exp \left(u m \int \Phi d\left(L_{m} \omega\right)\right) d P(\omega) \\
& =N^{m} \int_{\left\{\tau \in \Sigma \mid \operatorname{dist}\left(\Xi\left(L_{m}(\tau)\right), C\right) \leq r\right\}} \exp \left(m F\left(L_{m} \omega\right)\right) d P(\omega) \\
& =N^{m} \int_{\left\{\Xi L_{m} \in B(C, r)\right\}} \exp \left(m F\left(L_{m} \omega\right)\right) d P(\omega) \\
& =N^{m} \int_{\{\Xi \in B(C, r)\}} \exp (m F) d P_{m} \\
& =N^{m} Q_{m}(\{\Xi \in B(C, r)\}) \int \exp (m F) d P_{m} .
\end{aligned}
$$

Since the sequence $\left(P_{n}=P \circ L_{n}^{-1}\right)_{n} \subseteq \mathcal{P}\left(\mathcal{P}_{\mathrm{S}}(\Sigma)\right)$ has the large deviation property with respect to $(n)_{n}$ and rate function $I: \mathcal{P}_{\mathrm{S}}(\Sigma) \rightarrow \mathbb{R}$ given by $I(\mu)=\log N-h(\mu)$ where $h(\mu)$ denotes the entropy of $\mu$ (cf. [El]), it now 
follows from Theorem 3.1 that the sequence $\left(Q_{n}\right)_{n}$ has the large deviation property with respect to $(n)_{n}$ and rate function $(I-F)-\inf _{\nu \in \mathcal{P}_{\mathrm{S}}(\Sigma)}(I(\nu)-$ $F(\nu)$ ). It therefore follows from (3.2) and Theorem 3.1 that

$$
\begin{aligned}
\limsup _{m} & \frac{1}{m} \log \sum_{\substack{\sigma \in \Sigma^{m} \\
\operatorname{dist}(\Xi(L(\sigma)), C) \leq r}} s_{\sigma}^{u} \\
\leq & \log N+\limsup _{m} \frac{1}{m} \log Q_{m}(\{\Xi \in B(C, r)\}) \\
& +\limsup _{m} \frac{1}{m} \log \int \exp (m F) d P_{m} \\
\leq & \log N-\inf _{\substack{\mu \in \mathcal{P}_{\mathrm{S}}(\Sigma) \\
\Xi(\mu) \in B(C, r)}}\left((I(\mu)-F(\mu))-\inf _{\nu \in \mathcal{P}_{\mathrm{S}}(\Sigma)}(I(\nu)-F(\nu))\right) \\
& +\inf _{\nu \in \mathcal{P}_{\mathrm{S}}(\Sigma)}(I(\nu)-F(\nu)) \\
= & \log N+\sup _{\substack{\mu \in \mathcal{P}_{\mathrm{S}}(\Sigma) \\
\Xi(\mu) \in B(C, r)}}(F(\mu)-I(\mu)) \\
= & \sup _{\substack{\mu \in \mathcal{P}_{\mathrm{S}}(\Sigma) \\
\Xi(\mu) \in B(C, r)}}\left(u \int \Phi d \mu+h(\mu)\right) .
\end{aligned}
$$

This proves (3.1).

Next, write

$$
s=\sup _{\substack{\mu \in \mathcal{P}_{\mathrm{S}}(\Sigma) \\ \operatorname{dist}(\Xi(\mu), C) \leq r}}-\frac{h(\mu)}{\int \Phi d \mu}
$$

and let $t>s$. Put $\varepsilon=(t-s) / 3>0$. Hence, for all $\mu \in \mathcal{P}_{\mathrm{S}}(\Sigma)$ with $\operatorname{dist}(\Xi(\mu), C) \leq r$ we have $-h(\mu) / \int \Phi d \mu<s+\varepsilon=(s+2 \varepsilon)-\varepsilon$, and so

$$
(s+2 \varepsilon) \int \Phi d \mu+h(\mu) \leq \varepsilon \int \Phi d \mu \leq-\varepsilon c
$$

where $c=\log \left(1 / \max _{i} s_{i}\right)>0$. This and (3.1) imply that

$$
\begin{aligned}
\limsup _{m} \frac{1}{m} \log \sum_{\substack{\sigma \in \Sigma^{m} \\
\operatorname{dist}(\Xi(L(\sigma)), C) \leq r}} s_{\sigma}^{t} & \leq \limsup _{m} \frac{1}{m} \log \sum_{\substack{\sigma \in \Sigma^{m} \\
\operatorname{dist}(\Pi(\sigma), C) \leq r}} s_{\sigma}^{s+2 \varepsilon} \\
& \leq \sup _{\substack{\mu \in \mathcal{P}_{\mathrm{S}}(\Sigma) \\
\operatorname{dist}(\Xi(\mu), C) \leq r}}\left((s+2 \varepsilon) \int \Phi d \mu+h(\mu)\right) \\
& \leq-\varepsilon c .
\end{aligned}
$$


We therefore conclude that there exists an integer $M$ such that

$$
\sum_{\substack{\sigma \in \Sigma^{m} \\ \operatorname{dist}(\Xi(L(\sigma)), C) \leq r}} s_{\sigma}^{t} \leq e^{-m \varepsilon c / 2} \quad \text { for all } m \geq M .
$$

This gives the desired result.

THEOREM 3.4. Let $\mathbf{s}=\left(s_{0}, s_{1}, \ldots, s_{N-1}\right)$ be a finite list of numbers with $s_{i} \in(0,1)$. Let $X$ be a metric space and let $\Xi: \mathcal{P}(\Sigma) \rightarrow X$ be continuous with respect to the weak topology. If $C \subseteq X$ is a closed subset of $X$, then

$$
\limsup _{r \searrow 0} \delta_{\mathbf{S}}\left(\Delta_{r}(C)\right) \leq \sup _{\substack{\mu \in \mathcal{P}_{\mathrm{S}}(\Sigma) \\ \Xi(\mu) \in C}}-\frac{h(\mu)}{\sum_{i} \mu([i]) \log s_{i}} .
$$

Proof. Define $\Phi: \Sigma \rightarrow \mathbb{R}$ by $\Phi(\omega)=\log s_{\omega_{1}}$ for $\omega=\omega_{1} \omega_{2} \ldots \in \Sigma$. We first prove that

$$
\delta_{\mathbf{S}}\left(\Delta_{r}(C)\right) \leq \sup _{\substack{\mu \in \mathcal{P}_{\mathrm{S}}(\Sigma) \\ \operatorname{dist}(\Xi(\mu), C) \leq r}}-\frac{h(\mu)}{\int \Phi d \mu} \quad \text { for all } r \geq 0 .
$$

We therefore fix $r \geq 0$ and

$$
t>\sup _{\substack{\mu \in \mathcal{P}_{\mathrm{S}}(\Sigma) \\ \operatorname{dist}(\Xi(\mu), C) \leq r}}-\frac{h(\mu)}{\int \Phi d \mu} .
$$

It now follows from Proposition 3.3 that

$$
\sum_{m} \sum_{\substack{\omega \in \Sigma^{m} \\ \operatorname{dist}(\Xi(L(\omega)), C) \leq r}} s_{\omega}^{t}<\infty .
$$

This clearly implies that

$$
\begin{aligned}
\sum_{n \in \Delta_{r}(C)}\left(s_{d_{0}(n)} s_{d_{1}(n)} \ldots s_{d_{\gamma(n)}(n)}\right)^{t} & \\
& =\sum_{m} \sum_{\substack{k=N^{m}, \ldots, N^{m+1}-1 \\
k \in \Delta_{r}(C)}}\left(s_{d_{0}(k)} s_{d_{1}(k)} \ldots s_{d_{\gamma(k)}(k)}\right)^{t} \\
& \leq \sum_{m} \sum_{\substack{\omega \in \Sigma^{m+1} \\
\operatorname{dist}(\Xi(L(\omega)), C) \leq r}} s_{\omega}^{t}<\infty
\end{aligned}
$$

whence $\delta_{\mathbf{s}}\left(\Delta_{r}(C)\right) \leq t$. Since this holds for all $t$ as above, inequality (3.3) follows. 
It follows immediately from (3.3) that

$$
\limsup _{r \searrow 0} \delta_{\mathbf{s}}\left(\Delta_{r}(C)\right) \leq \inf _{k} \sup _{\substack{\mu \in \mathcal{P}_{\mathrm{S}}(\Sigma) \\ \operatorname{dist}(\Xi(\mu), C) \leq 1 / k}}-\frac{h(\mu)}{\int \Phi d \mu} .
$$

Finally, since the function $\Xi: \mathcal{P}(\Sigma) \rightarrow \Delta$ is continuous we conclude that $\Xi^{-1} B(C, 1 / k)$ is compact for all $k$. Hence, the set $C_{k}=\mathcal{P}_{\mathrm{S}}(\Sigma) \cap \Xi^{-1} B(C, 1 / k)$ is compact. Also, since the entropy function $h: \mathcal{P}(\Sigma) \rightarrow \mathbb{R}$ is upper semicontinuous (cf. [Wa, Theorem 8.2]), we deduce that the function $f: \mathcal{P}(\Sigma) \rightarrow \mathbb{R}$ given by $f(\mu)=-h(\mu) / \int \Phi d \mu$ is upper semicontinuous. Lemma 3.2 therefore implies that

$$
\inf _{k} \sup _{\mu \in C_{k}} f(\mu)=\sup _{\mu \in \bigcap_{k} C_{k}} f(\mu) \text {. }
$$

Since $\bigcap_{k} C_{k}=\bigcap_{k}\left(\mathcal{P}_{\mathrm{S}}(\Sigma) \cap \Xi^{-1} B(C, 1 / k)\right)=\mathcal{P}_{\mathrm{S}}(\Sigma) \cap \Xi^{-1} C$, combining (3.4) and (3.5) gives

$$
\limsup _{r \searrow 0} \delta_{\mathbf{S}}\left(\Delta_{r}(C)\right) \leq \inf _{k} \sup _{\substack{\mu \in \mathcal{P}_{\mathrm{S}}(\Sigma) \\ \operatorname{dist}(\Xi(\mu), C) \leq 1 / k}}-\frac{h(\mu)}{\int \Phi d \mu}=\sup _{\substack{\mu \in \mathcal{P}_{\mathrm{S}}(\Sigma) \\ \Xi(\mu) \in C}}-\frac{h(\mu)}{\int \Phi d \mu} .
$$

The desired result now follows since $\int \Phi d \mu=\sum_{i} \mu([i]) \log s_{i}$ for all probability measures $\mu$ on $\Sigma$.

Proof of Theorem 6 and the upper bound (1.13). Theorem 6 follows immediately from Theorem 3.4. We will now prove (1.13). Let $\mathbf{s}=\left(s_{0}, \ldots, s_{N-1}\right)$ $=(1 / N, \ldots, 1 / N)$, and observe that for this choice of $\mathbf{s}$ we have

$$
\sum_{n \in \Delta_{r}(C)} \frac{1}{n^{t}} \leq \sum_{n \in \Delta_{r}(C)} \frac{1}{N^{(\gamma(n)+1) t}}=\sum_{n \in \Delta_{r}(C)} s_{d(n)}^{t}
$$

for all $t \geq 0$ and all $r>0$, whence

$$
\begin{aligned}
\limsup _{r \searrow 0} \delta\left(\Delta_{r}(C)\right) & \leq \limsup _{r \searrow 0} \delta\left(\Delta_{r}(C) ; \mathbf{s}\right) \\
& \leq \sup _{\substack{\mu \in \mathcal{P}_{\mathcal{S}}(\Sigma) \\
\Xi(\mu) \in C}}-\frac{h(\mu)}{\sum_{i} \mu([i]) \log (1 / N)}=\sup _{\substack{\mu \in \mathcal{P}_{\mathcal{S}}(\Sigma) \\
\Xi(\mu) \in C}} \frac{h(\mu)}{\log N} .
\end{aligned}
$$

This proves (1.13).

4. Proof of the lower bound (1.14). Throughout this section $X$ will be a metric space and $\Xi: \mathcal{P}(\Sigma) \rightarrow X$ will be continuous with respect to the weak topology. Recall that $L: \Sigma^{*} \rightarrow \mathcal{P}(\Sigma)$ is defined as follows. For $\omega \in \Sigma^{*}$ we write $\bar{\omega}=\omega \omega \ldots$, and we let

$$
L(\omega)=\frac{1}{n} \sum_{i=0}^{n-1} \delta_{S^{k} \bar{\omega}} \quad \text { for } \omega \in \Sigma^{n} .
$$


For a subset $C \subseteq X, n \in \mathbb{N}$ and $r>0$, let

$$
\begin{aligned}
\Pi_{n}(C, r) & =\left\{\omega \in \Sigma^{n} \mid \operatorname{dist}(\Xi(L(\omega)), C) \leq r\right\}, \\
\Lambda(C) & =\lim _{r \searrow 0} \liminf _{n} \frac{\log \left|\Pi_{n}(C, r)\right|}{n \log N} .
\end{aligned}
$$

Lemma 4.1. Let $\mathcal{K}(X)=\{C \subseteq X \mid C$ is closed and non-empty $\}$ and equip $\mathcal{K}(X)$ with the Hausdorff metric $D$. Then the function $\Lambda: \mathcal{K}(X) \rightarrow \mathbb{R}$ is upper semicontinuous, i.e. for each $C \in \mathcal{K}(X)$ and $\varepsilon>0$, there exists $\delta>0$ such that

$$
\Lambda(K) \leq \Lambda(C)+\varepsilon \quad \text { for all } K \in \mathcal{K}(X) \text { with } D(K, C)<\delta .
$$

Proof. Let $C \in \mathcal{K}(X)$ and $\varepsilon>0$. Next, choose $r_{0}>0$ such that

$$
\liminf _{n} \frac{\log \left|\Pi_{n}(C, r)\right|}{n \log N}<\Lambda(C)+\varepsilon
$$

for $0<r \leq r_{0}$. Let $\delta=r_{0} / 2$. We now claim that $\Lambda(K) \leq \Lambda(C)+\varepsilon$ for all $K \in \mathcal{K}(X)$ with $D(K, C)<\delta$. To prove this, let $K \in \mathcal{K}(X)$ with $D(K, C)<\delta$. For all $0<r<\delta$ and all $n \in \mathbb{N}$ we have

$$
\Pi_{n}(K, r) \subseteq \Pi_{n}\left(C, r_{0}\right) .
$$

Indeed, first observe that $B\left(K, r_{0} / 2\right) \subseteq B\left(C, r_{0}\right)$ since $D(K, C)<\delta=r_{0} / 2$. Hence, if $\omega \in \Pi_{n}(K, r)$, then $\Xi(L(\omega)) \in B(K, r) \subseteq B\left(K, r_{0} / 2\right) \subseteq B\left(C, r_{0}\right)$. This proves (4.1). It follows from (4.1) that

$$
\liminf _{n} \frac{\log \left|\Pi_{n}(K, r)\right|}{n \log N} \leq \liminf _{n} \frac{\log \left|\Pi_{n}\left(C, r_{0}\right)\right|}{n \log N}<\Lambda(C)+\varepsilon
$$

for all $0<r<\delta$, whence

$$
\Lambda(K)=\lim _{r \searrow 0} \liminf _{n} \frac{\log \left|\Pi_{n}(K, r)\right|}{n \log N} \leq \Lambda(C)+\varepsilon .
$$

We will equip $\Sigma$ with the metric $d_{\Sigma}(\omega, \sigma)=N^{-\sup \{n|\omega| n=\sigma \mid n\}}$. In the results below (Lemma 4.2 and Proposition 4.4) we will always compute the Hausdorff dimension of a subset of $\Sigma$ with respect to the metric $d_{\Sigma}$.

Lemma 4.2. Let $C \subseteq X$ be a closed subset of $X$. Then

$$
\operatorname{dim}\left\{\omega \in \Sigma \mid \lim _{n} \operatorname{dist}(\Xi(L(\omega \mid n)), C)=0\right\} \leq \Lambda(C) .
$$

(Recall that dim denotes the Hausdorff dimension.)

Proof. For a subset $E$ of $\Sigma$, we let $\operatorname{dim}_{\mathrm{B}} E$ denote the lower box dimension of $E$; the reader is referred to [Ed] or [Fa1] for the definition. We will use the fact that $\operatorname{dim} E \leq \underline{\operatorname{dim}}_{\mathrm{B}} E$ for all $E \subseteq \Sigma$ (cf. [Ed]). Write $M=\{\omega \in \Sigma \mid$ $\left.\lim _{n} \operatorname{dist}(\Xi(L(\omega \mid n)), C)=0\right\}$. Also, for $n \in \mathbb{N}$ and $r>0$, write

$$
M_{n}(r)=\{\omega \in \Sigma \mid \operatorname{dist}(\Xi(L(\omega \mid k)), C) \leq r \text { for } k \geq n\} .
$$


We clearly have $M \subseteq \bigcup_{n} M_{n}(r)$ for all $r>0$, whence

$$
\operatorname{dim} M \leq \sup _{n} \operatorname{dim} M_{n}(r) \leq \sup _{n} \underline{\operatorname{dim}}_{\mathrm{B}} M_{n}(r)
$$

for all $r>0$. However, it is easily seen that $M_{n}(r) \subseteq \bigcup_{\omega \in \Pi_{k}(C, r)}[\omega]$ for $k \geq n$. Hence, for $k \geq n$, the family $([\omega])_{\omega \in \Pi_{k}(C, r)}$ is a covering of $M_{n}(r)$ of sets with $\operatorname{diam}([\omega])=N^{-k}$. This implies that

$$
\underline{\operatorname{dim}}_{\mathrm{B}} M_{n}(r) \leq \liminf _{k \geq n} \frac{\log \left|\Pi_{k}(C, r)\right|}{k \log N} .
$$

Combining (4.2) and (4.3) gives

$$
\operatorname{dim} M \leq \liminf _{k} \frac{\log \left|\Pi_{k}(C, r)\right|}{k \log N} \quad \text { for all } r>0 .
$$

This completes the proof.

Lemma 4.3. Let $\mu \in \mathcal{P}_{\mathrm{S}}(\Sigma)$ with $\operatorname{supp} \mu=\Sigma$. (Here $\operatorname{supp} \mu$ denotes the topological support of $\mu$.) Then there exists a sequence $\left(\mu_{n}\right)_{n}$ of probability measures on $\Sigma$ such that:

(1) $\mu_{n} \rightarrow \mu$ weakly.

(2) For each $n$, the measure $\mu_{n}$ is a Gibbs state for a Hölder continuous function. In particular, $\mu_{n}$ is ergodic and $\operatorname{supp} \mu_{n}=\Sigma$ for all $n$.

(3) $h\left(\mu_{n}\right) \rightarrow h(\mu)$.

Proof. This lemma is proved in [Ol2] and the proof is therefore omitted.

If $\mu$ is a probability measure on $\Sigma$, we define the upper Hausdorff dimension of $\mu$ by

$$
\overline{\operatorname{dim}} \mu=\inf _{\mu(E)=1} \operatorname{dim} E .
$$

(Recall that dim denotes the Hausdorff dimension.) The next result provides a formula for the upper Hausdorff dimension of an ergodic probability measure on $\Sigma$. This result is well known and follows easily from the Shannon-MacMillan-Breiman theorem. However, for sake of completeness we have included the short proof.

Proposition 4.4. Let $\mu$ be an ergodic probability measure on $\Sigma$. Then

$$
\overline{\operatorname{dim}} \mu=\frac{h(\mu)}{\log N} .
$$

Proof. Since $\mu$ is ergodic, it follows from the Shannon-MacMillan-Breiman theorem that

$$
\frac{\log \mu([\omega \mid n])}{n} \rightarrow-h(\mu) \quad \text { for } \mu \text {-a.a. } \omega \in \Sigma .
$$


Clearly $B\left(\omega, N^{-n}\right)=[\omega \mid n]$ for all $\omega \in \Sigma$ and $n \in \mathbb{N}$, and therefore

$$
\frac{\log \mu\left(B\left(\omega, N^{-n}\right)\right)}{\log N^{-n}}=\frac{\log \mu([\omega \mid n])}{-n \log N} \rightarrow \frac{h(\mu)}{\log N} \quad \text { for } \mu \text {-a.a. } \omega \in \Sigma .
$$

The desired result follows from this and the fact that

$$
\overline{\operatorname{dim}} \mu=\underset{\omega}{\operatorname{ess} \sup } \frac{\log \mu\left(B\left(\omega, N^{-n}\right)\right)}{\log N^{-n}}
$$

(cf. [Fa2]).

Proposition 4.5. Let $C$ be a closed subset of $X$. Then

$$
\sup _{\substack{\mu \in \mathcal{P}_{\mathrm{S}}(\Sigma) \\ \Xi(\mu) \in C}}-\frac{h(\mu)}{\log N} \leq \Lambda(C) .
$$

Proof. Fix $\mu \in \mathcal{P}_{\mathrm{S}}(X)$ with $\Xi \mu \in C$ and $\varepsilon>0$. It follows from Lemma 4.1 that the function $\mathcal{K}(X) \rightarrow \mathbb{R}: K \mapsto \Lambda(K)$ is upper semicontinuous with respect to the Hausdorff metric $D$, and we can therefore choose $\delta_{\varepsilon}>0$ such that

$$
\Lambda(K) \leq \Lambda(C)+\varepsilon \quad \text { for all } K \in \mathcal{K}(X) \text { with } D(K, C)<\delta_{\varepsilon} .
$$

Next, choose an $S$-invariant measure $\nu$ on $\Sigma$ such that $\operatorname{supp} \nu=\Sigma$ and let $\mu_{r}=(1-r) \mu+r \nu \in \mathcal{P}_{\mathrm{S}}(\Sigma)$ for $r \in(0,1)$. As $\Xi$ is continuous and $\Xi(\mu) \in C$ there exists $0<r_{\varepsilon}<1$ such that

$$
\operatorname{dist}\left(\Xi \mu_{r}, C\right)<\delta_{\varepsilon} / 2 \quad \text { for all } 0<r<r_{\varepsilon} .
$$

Fix $0<r<r_{\varepsilon}$. It follows from Lemma 4.3 and the continuity of $\Xi$ that we may choose a sequence $\left(\mu_{r, n}\right)_{n}$ of ergodic $S$-invariant probability measures on $\Sigma$ such that $\mu_{r, n} \rightarrow \mu_{r}$ weakly, $\operatorname{supp} \mu_{r, n}=\Sigma$ for all $n$, $h\left(\mu_{r, n}\right) \rightarrow h\left(\mu_{r}\right)$ and $\operatorname{dist}\left(\Xi \mu_{r, n}, C\right)<\delta_{\varepsilon}$.

Define $L_{m}: \Sigma \rightarrow \mathcal{P}_{\mathrm{S}}(\Sigma)$ as in the proof of Proposition 3.3, i.e. $L_{m}(\omega)=$ $(1 / m) \sum_{k=0}^{m-1} \delta_{S^{k} \omega}$. It follows from the ergodicity of $\mu_{r, n}$ and the ergodic theorem that $\mu_{r, n}\left(\left\{\omega \in \Sigma \mid \lim _{m} L_{m} \omega=\mu_{r, n}\right\}\right)=1$. Hence

$$
\begin{aligned}
\overline{\operatorname{dim}} \mu_{r, n} & \leq \operatorname{dim}\left\{\omega \in \Sigma \mid \lim _{m} L_{m} \omega=\mu_{r, n}\right\} \\
& \leq \operatorname{dim}\left\{\omega \in \Sigma \mid \lim _{m} \operatorname{dist}\left(\Xi L_{m} \omega, C \cup\left\{\Xi \mu_{r, n}\right\}\right)=0\right\} \\
& \leq \Lambda\left(C \cup\left\{\Xi \mu_{r, n}\right\}\right) \leq \Lambda(C)+\varepsilon,
\end{aligned}
$$

where the last inequality in (4.7) follows from (4.5) and (4.6).

Also, since $\mu_{r, n}$ is ergodic, it follows from Proposition 4.4 that

$$
\overline{\operatorname{dim}} \mu_{r, n}=-\frac{h\left(\mu_{r, n}\right)}{\log N} .
$$


Finally, the properties of the sequence $\left(\mu_{r, n}\right)_{n}$ imply that

$$
-\frac{h\left(\mu_{r, n}\right)}{\log N} \rightarrow-\frac{h\left(\mu_{r}\right)}{\log N}
$$

Combining (4.7)-(4.9) and using the fact that the entropy map $h$ : $\mathcal{P}_{\mathrm{S}}(\Sigma) \rightarrow \mathbb{R}$ is affine (cf. [Wa]) we conclude that

$-\frac{(1-r) h(\mu)+r h(\nu)}{\log N}=-\frac{h\left(\mu_{r}\right)}{\log N} \leq \Lambda(C)+\varepsilon \quad$ for all $\varepsilon>0$ and $0<r<r_{\varepsilon}$.

Letting first $r \searrow 0$ and then $\varepsilon \searrow 0$ gives $-h(\mu) / \log N \leq \Lambda(C)$. Since $\mu \in \mathcal{P}_{\mathrm{S}}(X)$ with $\Xi(\mu) \in C$ was arbitrary, this yields the desired result.

Theorem 4.6. Let $C$ be a closed subset of $X$. Then

$$
\Lambda(C) \leq \liminf _{r \searrow 0} \underline{\operatorname{dim}}\left(\Delta_{r}(C)\right) .
$$

Proof. Fix $\varepsilon>0$. We can thus choose $r_{0}>0$ such that

$$
\Lambda(C)-\varepsilon<\liminf _{n} \frac{\log \left|\Pi_{n}(C, r)\right|}{n \log N} \quad \text { for all } 0<r<r_{0} .
$$

Hence, for each $0<r<r_{0}$ there exists a positive integer $n_{r}$ such that

$$
N^{n(\Lambda(C)-\varepsilon)} \leq\left|\Pi_{n}(C, r)\right| \quad \text { for all } n \geq n_{r} .
$$

Next, for each $n \in \mathbb{N}$, let $k_{n}$ denote the unique integer with $N^{k_{n}} \leq n<$ $N^{k_{n}+1}$. It clearly follows that

$$
N_{n}\left(\Delta_{r}(C)\right) \geq N_{N^{k_{n}}}\left(\Delta_{r}(C)\right) \geq\left|\Pi_{k_{n}}(C, r)\right| .
$$

Hence, for all $0<r<r_{0}$ and $n \geq N^{n_{r}}$ (and so $k_{n} \geq n_{r}$ ), inequalities (4.10) and (4.11) imply that

$$
\frac{\log N_{n}\left(\Delta_{r}(C)\right)}{\log n} \geq \frac{\log \left|\Pi_{k_{n}}(C, r)\right|}{\log N^{k_{n}+1}} \geq \frac{\log N^{k_{n}(\Lambda(C)-\varepsilon)}}{\log N^{k_{n}+1}}=(\Lambda(C)-\varepsilon) \frac{k_{n}}{k_{n}+1} .
$$

This shows that $\underline{\operatorname{dim}} \Delta_{r}(C)=\liminf _{n} \frac{\log \left|N_{n}\left(\Delta_{r}(C)\right)\right|}{\log n} \geq \Lambda(C)-\varepsilon$ for all $0<r<r_{0}$, whence $\liminf _{r \searrow 0} \underline{\operatorname{dim}} \Delta_{r}(C) \geq \Lambda(C)-\varepsilon$. Finally, letting $\varepsilon \searrow 0$ completes the proof.

Proof of the lower bound (1.14). The lower bound (1.14) follows immediately from Proposition 4.5 and Theorem 4.6.

5. Proof of Theorem 7. We begin with a small lemma that allows us to consider only $N$-ary primitive integers (cf. (1.18)).

Lemma 5.1. Let $X$ be a metric space and let $\Xi: \mathcal{P}(\Sigma) \rightarrow X$ be continuous with respect to the weak topology. Let $C$ be a closed subset of $X$ and 
$r>0$. Let

$$
n=\sum_{i=0}^{\gamma(n)} d_{i}(n) N^{i}
$$

and $k \in \mathbb{N} \cup\{0\}$, and write

$$
m=\sum_{j=0}^{k} \sum_{i=0}^{\gamma(n)} d_{i}(n) N^{i+j}
$$

i.e. $m$ is the unique integer such that $d(m)=\underbrace{d(n) \ldots d(n)}_{k+1 \text { times }}$. If $n \in \Delta_{r}(C)$, then $m \in \Delta_{r}(C)$.

Proof. Recall definition (1.7) of the measures $I(m)$ and $I(n)$. We clearly have

$$
\begin{aligned}
I(m) & =\frac{1}{\gamma(m)+1} \sum_{i=0}^{\gamma(m)} \delta_{S^{i}(\overline{d(m)})} \\
& =\frac{1}{(k+1)(\gamma(n)+1)}(k+1) \sum_{i=0}^{\gamma(n)} \delta_{S^{i}(\overline{d(n)})}=I(n) .
\end{aligned}
$$

The desired result follows from this.

Proof of Theorem 7. (i) It follows from Lemma 5.1 that

$$
\sum_{n \in \Delta_{r}(C)} \frac{1}{\gamma(n)+1} s_{d(n)}^{t}=\sum_{n \in \Delta_{r}(C) \cap \mathbb{P}} \sum_{k=1}^{\infty} \frac{1}{k(\gamma(n)+1)} \underbrace{t}_{k \text { times }} \underbrace{t}_{d(n) \ldots d(n)} .
$$

This implies that

$$
\begin{aligned}
Z_{\Delta_{r}(C)}(t ; \mathbf{s}) & =\sum_{n \in \Delta_{r}(C)} \frac{1}{\gamma(n)+1} s_{d(n)}^{t} \\
& =\sum_{n \in \Delta_{r}(C) \cap \mathbb{P}} \sum_{k=1}^{\infty} \frac{1}{k(\gamma(n)+1)} s_{k \text { times }}^{t} \underbrace{}_{n \in \Delta_{r}(C) \cap \mathbb{P}} \frac{1}{\gamma(n)+1} \sum_{k=1}^{\infty} \frac{1}{k} s_{d(n)}^{t k} \\
& =\sum_{n \in \Delta_{r}(C) \cap \mathbb{P}} \frac{1}{\gamma(n)+1} \log \left(\frac{1}{1-s_{d(n)}^{t}}\right) \\
& =\log \prod_{n \in \Delta_{r}(C) \cap \mathbb{P}}\left(\frac{1}{1-s_{d(n)}^{t}}\right)^{1 /(\gamma(n)+1)} .
\end{aligned}
$$


(ii) For $k \in \mathbb{N}$ write $a_{k}=|\{n \in \mathbb{N} \mid \gamma(n)+1=k\}|$. We now have

$$
\zeta_{\Delta_{r}(C)}(t ; \mathbf{s})=\sum_{\Delta_{r}(C)} s_{d(n)}^{t}=\sum_{k=1}^{\infty} \sum_{\substack{n \in \Delta_{r}(C) \\ \gamma(n)+1=k}} s_{d(n)}^{t}=\sum_{k=1}^{\infty} a_{k} s^{t k},
$$

and

$$
\begin{aligned}
Z_{\Delta_{r}(C)}(t ; \mathbf{s}) & =\sum_{\Delta_{r}(C)} \frac{1}{\gamma(n)+1} s_{d(n)}^{t} \\
& =\sum_{k=1}^{\infty} \sum_{\substack{n \in \Delta_{r}(C) \\
\gamma(n)+1=k}} \frac{1}{\gamma(n)+1} s_{d(n)}^{t}=\sum_{k=1}^{\infty} \frac{1}{k} a_{k} s^{t k}
\end{aligned}
$$

Hence

$$
\frac{d}{d t} Z_{\Delta_{r}(C)}(t ; \mathbf{s})=\sum_{k=1}^{\infty} a_{k} s^{t k} \log s=\zeta_{\Delta_{r}(C)}(t ; \mathbf{s}) \log s .
$$

This completes the proof.

\section{References}

[Ap] T. Apostol, Modular Functions and Dirichlet Series in Number Theory, Grad. Texts in Math. 41, Springer, New York, 1976.

[BaT1] M. T. Barlow and S. J. Taylor, Fractional dimension of sets in discrete spaces. With a reply by J. Naudts, J. Phys. A 22 (1989), 2621-2628.

[BaT2] - - Defining fractal subsets of $\mathbb{Z}^{d}$, Proc. London Math. Soc. 64 (1992), $125-152$.

[BeF] T. Bedford and A. Fisher, Analogues of the Lebesgue density theorem for fractal sets of reals and integers, ibid. 64 (1992), 95-124.

[BS] R. Bellman and H. Shapiro, On a problem in additive number theory, Ann. of Math. 49 (1948), 333-340.

[Be] A. Besicovitch, On the sum of digits of real numbers represented in the dyadic system, Math. Ann. 110 (1934), 321-330.

[BlK] R. Blei and T. Körner, Combinatorial dimension and random sets, Israel J. Math. 47 (1984), 65-74.

[Bu] L. E. Bush, An asymptotic formula for the average sum of the digits of integers, Amer. Math. Monthly 47 (1940), 154-156.

[Co] J. Coquet, Power sums of digital sums, J. Number Theory 22 (1986), 161-176.

[De] H. Delange, Sur la fonction sommatoire de la fonction "somme des chiffres", Enseign. Math. 21 (1975), 31-47.

[DZ] A. Dembo and O. Zeitouni, Large Deviations Techniques and Applications, Jones and Bartlett Publishers, Boston, MA, 1993.

[DT1] J.-M. Dumont and A. Thomas, Digital sum moments and substitutions, Acta Arith. 64 (1993), 205-225.

[DT2] - - , Modifications de nombres normaux par des transducteurs, ibid. 68 (1994), 153-170. 
[DT3] J.-M. Dumont and A. Thomas, Gaussian asymptotic properties of the sum-ofdigits function, J. Number Theory 62 (1997), 19-38.

[Ed] G. Edgar, Integral, Probability, and Fractal Measures, Springer, New York, 1998.

[Eg] H. G. Eggleston, The fractional dimension of a set defined by decimal properties, Quart. J. Math. Oxford Ser. 20 (1949), 31-36.

[El] R. Ellis, Entropy, Large Deviations, and Statistical Mechanics, Grundlehren Math. Wiss. 271, Springer, New York, 1985.

[Fa1] K. J. Falconer, Fractal Geometry-Mathematical Foundations and Applications, Wiley, 1990.

[Fa2] -, Techniques in Fractal Geometry, Wiley, Chichester, 1997.

[FFW] A.-H. Fan, D.-J. Feng and J. Wu, Recurrence, dimension and entropy, preprint, 2001.

[Fu] H. Furstenberg, Intersections of Cantor sets and transversality of semigroups, in: Problems in Analysis (Sympos. Salomon Bochner, 1969), Princeton Univ. Press, Princeton, NJ, 1970, 41-59.

[GKPT] P. Grabner, P. Kirschenhofer, H. Prodinger and R. Tichy, On the moments of the sum-of-digits function, in: Applications of Fibonacci Numbers 5, Kluwer, Dordrecht, 1993, 263-271.

[KC] R. Kennedy and C. Coope, An extension of a theorem by Cheo and Yien concerning digital sums, Fibonacci Quart. 29 (1991), 145-149.

[K] P. Kirschenhofer, On the variance of the sum of digits function, in: Numbertheoretic Analysis (Vienna, 1988-89), Lecture Notes in Math. 1452, Springer, Berlin, 1990, 112-116.

$[\mathrm{LvF}] \quad$ M. Lapidus and M. van Frankenhuysen, Fractal Geometry and Number Theory. Complex Dimensions of Fractal Strings and Zeros of Zeta Functions, Birkhäuser, Boston, MA, 2000.

[NS] Y. Nakai and I. Shiokawa, Normality of numbers generated by the values of polynomials at primes, Acta Arith. 81 (1997), 345-356.

[Na] J. Naudts, Dimension of discrete fractal spaces, J. Phys. A 21 (1988), 447-452.

[Ol1] L. Olsen, Multifractal analysis of divergence points of deformed measure theoretical Birkhoff averages, preprint, 2001.

[Ol2] -, Distribution of digits in integers: Besicovitch-Eggleston subsets of $\mathbb{N}$, preprint, 2001.

[PW] Y. Pesin and H. Weiss, The multifractal analysis of Birkhoff averages and large deviations, preprint, 1999.

[Sh] I. Shiokawa, Asymptotic distributions of digits in integers, in: Number Theory, Vol. I (Budapest, 1987), Colloq. Math. Soc. János Bolyai 51, North-Holland, Amsterdam, 1990, 505-525.

[Va] S. R. S. Varadhan, Asymptotic probabilities and differential equations, Comm. Pure Appl. Math. 19 (1966), 261-286.

[Wa] P. Walters, An Introduction to Ergodic Theory, Springer, 1982.

Department of Mathematics

University of St. Andrews

St. Andrews, Fife KY16 9SS, Scotland

E-mail: lo@st-and.ac.uk 